\title{
Temperamento, neuroticismo e auto-estima: estudo preliminar
}

\section{Temperament, neuroticism and self-esteem: preliminary study}

\author{
Patrícia do Carmo Pereira ITO \\ Mônica GOBITTA2,4 \\ Raquel Souza Lobo GUZZO2,3
}

\begin{abstract}
Resumo
Esta pesquisa investigou correlações entre temperamento, neuroticismo e auto-estima. Participaram da amostra 42 universitários na faixa etária dos 19 aos 21 anos, os quais responderam às Escalas Fatorial de Ajustamento Emocional/Neuroticismo, Pavlovian Temperament Survey e Auto-Estima de Rosenberg. Os resultados indicaram que a dimensão força de excitação (Pavlovian Temperament Survey) apresentou correlações negativas significativas com as dimensões vulnerabilidade e ansiedade (Escalas Fatorial de Ajustamento Emocional/Neuroticismo); a força de inibição (Pavlovian Temperament Survey) apresentou correlação negativa significativa com a ansiedade da Escalas Fatorial de Ajustamento Emocional/Neuroticismo e a mobilidade (Pavlovian Temperament Survey) se correlacionou positivamente com desajustamento psicossocial (Escalas Fatorial de Ajustamento Emocional/Neuroticismo). No que se refere à correlação entre Pavlovian Temperament Survey e Escala de Auto-Estima, não foram obtidas correlações significativas. A correlação entre Escalas Fatorial de Ajustamento Emocional/Neuroticismo e autoestima apresentou correlações negativas significativas nas dimensões vulnerabilidade, ansiedade e depressão. Esses resultados, importantes na elaboração e implantação de programas de prevenção e intervenção, permitem verificar como diferentes características de personalidade se relacionam e que perfil individual predispõem.
\end{abstract}

Unitermos: auto-estima; desenvolvimento humano; neuroticismo; personalidade.

\begin{abstract}
This research investigated the possible correlation among temperament, neuroticism and self-esteem. The sample was composed by 42 students from 19 to 21 years, who answered the Emotional Adjustment /Neuroticism Factorial Scales, Pavlovian Temperament Survey and Rosenberg's Self-esteem. Obtained results indicated significant negative correlation between the dimension Strength of Excitement (Pavlovian Temperament Survey) and the dimensions vulnerability and anxiety (Emotional Adjustment/Neuroticism Factorial Scales), significant negative correlation between the Strength of Inhibition (Pavlovian Temperament Survey) and the anxiety of Emotional Adjustment/Neuroticism Factorial Scales, and positive correlation between the Mobility (Pavlovian Temperament Survey) maladjustment psychosocial (Emotional Adjustment/Neuroticism Factorial Scales). There was no significant correlation between Pavlovian Temperament Survey and Self-esteem Scale. The correlation between Emotional Adjustment/Neuroticism Factorial and Self-esteem Scales presented significant negative correlation in vulnerability, anxiety and depression dimensions. These results are important to the development and implantation of intervention and prevention programs. Also, they bring up the different personality characteristics.
\end{abstract}

Uniterms: self esteem; human development; neuroticism; personality.

\section{४ $\mathbf{\nabla} \boldsymbol{\nabla}$}

1 Faculdade de Americana, Curso de Psicologia. Americana, SP, Brasil.

2 Pontifícia Universidade Católica de Campinas, Centro de Ciências da Vida, Faculdade de Psicologia. Av. Jonh Boyd Dunlop, s/n., Prédio Administrativo, Jardim Ipaussurama, 13060-904, Campinas, SP, Brasil. Correspondência para/Correspondence to: M. GOBITTA. E-mail: <mgobitta@sigmanet.com.br>.

3 Pontifícia Universidade Católica de Campinas, Centro de Ciências da Vida, Programa de Pós-Graduação em Psicologia. Campinas, SP, Brasil.

4 Centro Universitário Salesiano de São Paulo, Pós-Graduação Latu Sensu de Educação Social e Psicopedagogia. Campinas, SP, Brasil. 
Teorias e dados de pesquisas atuais apóiam a visão de que o desenvolvimento é o resultado de múltiplos fatores, que incluem características individuais, interações face a face e fatores do contexto. Nesse sentido, Teglasi (1998) destaca como fatores importantes: a) fatores psicológicos: que incluem pensamentos relacionados à importância e à implicação de eventos, sentimentos e repertório de comportamentos de enfrentamento; b) fatores biológicos: relacionados às respostas fisiológicas que influenciam processos psicológicos, as quais se traduzem em estados subjetivos de ansiedade, depressão, irritabilidade, inquietação, pensamentos recorrentes ou dificuldades de concentração e c) grau de apoio social: pessoas e recursos do contexto que o indivíduo dispõe para manter ou restabelecer o equilíbrio emocional, dos pensamentos, sentimentos e comportamentos.

De acordo com Brito e Koller (1999), o desenvolvimento adaptado é determinado pela rede de apoio social, ou seja, os recursos externos de apoio que proporcionam reforço às estratégias de enfrentamento das situações de vida; pela rede de apoio afetivo, o desenvolver-se em um ambiente coeso e sem conflito no ambiente familiar; e também pelas características individuais, como autonomia, auto-estima e orientação social positiva.

Segundo essas autoras, a possibilidade de se desenvolver adaptativamente e de dispor de recursos que incrementem os determinantes acima protege a pessoa de doenças e sintomas psicopatológicos, mesmo quando ela está frente a situações adversas. Então, a pessoa que apresenta um desenvolvimento saudável, ou seja, que apresenta características individuais que revelam sua capacidade emocional e social para a adaptação, vive em um ambiente afetivo, utiliza estratégias eficazes para lidar com conflitos ou situações de estresse e conta com uma rede de apoio social e afetiva efetiva é definida como resiliente.

Szymanski e Yunes (2001) relatam que a abordagem que melhor se aproxima do fenômeno da resiliência e oferece um modelo explicativo dos processos desenvolvimentais é a Abordagem Ecológica de Urie Bonfenbrenner. As autoras descrevem que "a abordagem ecológica procura não só descrever e explicar os efeitos do ecossistema do indivíduo, mas também busca oferecer subsídios para a elaboração de

144 programas de intervenção social" (p.42).
Nessa perspectiva, Bronfenbrenner (1995) considera que o desenvolvimento humano ocorre por meio de processos progressivamente mais complexos de interações recíprocas entre um organismo humano biopsicológico ativo e pessoas, objetos e símbolos do ambiente imediato. Esse organismo biopsicológico inclui características relacionadas ao temperamento e à personalidade, constituição neurológica e bioquímica, funcionamento cognitivo na infância e posteriormente medidas de inteligência, realização acadêmica, autoconceito, papel das expectativas, auto-eficácia, valores e metas.

O desenvolvimento humano para Bronfenbrenner (1995) resulta da interação de quatro dimensões, o chamado Modelo PPCT, onde o primeiro $P$ é atribuído ao processo proximal: as formas duradouras de interação no ambiente imediato do indivíduo (interações face a face). É importante destacar que as interações são afetadas pelas características da criança e da outra pessoa envolvida nessa interação e também pela natureza dos contextos onde ocorre; o contínuo processo de interação proximal é o aspecto mais importante na formação dos aspectos estáveis do desenvolvimento do indivíduo.

O segundo $\mathrm{P}$ diz respeito à dimensão pessoa, ou seja, o próprio indivíduo que recebe o impacto da experiência está relacionado também às características biopsicológicas, tais como temperamento e personalidade, constituição neurológica e bioquímica do sistema nervoso central, funcionamento cognitivo (comportamento de atenção, rapidez de resposta, níveis de atividade e grau de auto-regulação), autoconceito, auto-estima, auto-eficácia e valores.

A terceira dimensão considerada é o C de contexto, que inclui os contextos de desenvolvimento do indivíduo, os quais são compostos de estruturas denominadas microssistema, mesossistema, exossistema e macrossistema.

O microssistema representa o ambiente onde ocorrem as situações de interação nas quais a criança está em contato com outra pessoa (pais, professores, vizinhos, parentes, amigos mais próximos). Bronfenbrenner atribui a esse sistema, basicamente, a família, a escola e o grupo social e salienta que o seu ponto característico é a sua natureza bidirecional, na qual um influencia o outro. A qualidade das interações 
no microssistema deve ser positiva para um desenvolvimento saudável e para a formação de fatores de proteção do indivíduo.

O próximo sistema, o mesossitema, é uma composição de microssistemas associados, e representa a interação entre eles. O outro sistema considerado por Bronfenbrenner é o exossistema, que são locais que não envolvem a criança diretamente, mas incluem as interações daqueles que têm um relacionamento com a criança, são os contextos que diretamente afetam pessoas que têm relações próximas com o indivíduo. 0 nível mais amplo do contexto de desenvolvimento é o macrossistema, que se refere ao nível político e cultural de influências nos outros níveis do sistema dentro dos quais a criança é participante. Valores culturais e idéias acerca da infância fornecem diretrizes para os cuidados com crianças.

Um outro aspecto do modelo ecológico diz respeito ao $T$ de tempo; essa dimensão é também denominada por cronossistema. Para Bronfenbrenner (1995, p.641) "o desenvolvimento do curso da vida do indivíduo é visto como embutido e fortemente modelado por condições e eventos que ocorrem durante o período histórico através do qual a pessoa vive".

Considerando as dimensões postuladas pela abordagem ecológica, inúmeras pesquisas têm sido desenvolvidas relacionadas aos fatores que influenciam o desenvolvimento do indivíduo. Muitas delas têm-se voltado ao estudo das características biopsicológicas, as quais incluem características relacionadas à personalidade e ao temperamento e que no modelo PPCT de Bronfenbrenner estariam presentes na dimensão Pessoa.

Temperamento e personalidade são duas características individuais que apresentam um vocabulário descritivo comum (Teglasi, 1995), mas que podem ser diferenciadas claramente com base em cinco aspectos: 1) o temperamento é biologicamente determinado, e a personalidade é um produto do ambiente social; 2) os traços temperamentais podem ser identificados desde cedo na criança, e a personalidade é compartilhada em períodos posteriores do desenvolvimento; 3) diferenças individuais nos traços de temperamento, como ansiedade, extroversão, introversão e busca de estimulação são também observadas em animais, enquanto a personalidade é prerrogativa do humano; 4) o temperamento apresenta aspectos estilísticos, se referindo a características formais de comportamento, já a personalidade contém aspectos relativos a conteúdos do comportamento; 5) ao contrário do temperamento, que se refere principalmente a traços ou mecanismos, a personalidade está relacionada ao funcionamento integrativo do comportamento humano (Strelau, 1998).

Nas várias teorias de temperamento (Buss, 1995; Chess \& Thomas, 1987; Clark \& Watson, 1999; Eysenck, 1974; Goldsmith et al., 1987; Rothbart, 1986), esse construto tem sido caracterizado como a expressão prematura de diferenças individuais na personalidade, o fundamento biológico no qual a personalidade se estrutura. Como bem descreve Graziano, Jensen-Campbell e Sullivan-Logan (1998), o temperamento é o núcleo em torno do qual a personalidade se desenvolve.

A esse respeito, na década de 1990 e início de 2000, ganhou destaque a discussão realizada sobre a relação dos fatores de personalidade incluídos no Big Five, os Cinco Grandes Fatores (CGF) (Digman, 1990; Hutz et al., 1998) e o temperamento. Vários fatores relacionados pelos Cinco Grandes Fatores (neuroticismo, extroversão, realização, socialização e abertura para experiência) são também relacionados como dimensões ou traços de temperamento.

Nesse sentido, Rothbart e Ahadi (1994) afirmam que as dimensões de temperamento que emergem da pesquisa com bebês e crianças pequenas são similares às estruturas de personalidade, encontradas nos estudos dos Cinco Grandes Fatores, realizadas em diferentes culturas com adultos.

Uma abordagem contemporânea que tem realizado estudos sobre as relações entre temperamento e personalidade é a Teoria Regulativa do Temperamento desenvolvida por Jan Strelau, a qual considera que o temperamento refere-se a"traços básicos, relativamente estáveis, expressos principalmente nas características formais de reações e comportamento. Esses traços estão presentes desde cedo na criança e podem ser encontrados em animais. Primariamente determinado por mecanismos de origem biológica, o temperamento está sujeito a mudanças causadas pela maturação e pela interação entre indivíduo e genótipo específico - ambiente" (Strelau, 1998, p.165). 
Nessa abordagem do temperamento, um dos instrumentos utilizados para avaliação das características temperamentais é a escala Pavlovian Temperament Survey (PTS), desenvolvida com o objetivo de avaliar a expressão comportamental das propriedades do sistema nervoso central, conforme entendido por Pavlov. Tais propriedades foram caracterizadas por Pavlov, do ponto de vista funcional e não fisiológico, reforçando o papel desempenhado por elas no processo de adaptação do indivíduo ao ambiente e referem-se à Força de Excitação (FE), Força de Inibição (FI) e Mobilidade (MO) (Strelau, Angleitner \& Newberry, 1999).

Pesquisas realizadas com esses e outros instrumentos para avaliação do temperamento objetivam investigar a relação entre características de temperamento e personalidade, desenvolvimento ajustado, interação entre pares e também considerando o temperamento como um dos fatores responsáveis pela resiliência.

A esse respeito, Losel e Bliesener (1994) em sua pesquisa obtiveram dados que indicam que os indivíduos resilientes são caracterizados por serem mais inteligentes e mais flexíveis no temperamento, apresentam autoconceito positivo, percebem-se como capazes e orientados para realização, tendendo a ser mais ativos.

Em outro trabalho, Hertzig (1994), comparando dados obtidos no estudo de resiliência conduzido por Werner em crianças em situação de risco de Kauai, constatou que algumas características individuais, da família e do círculo social contribuíam para a resiliência dessas crianças. Como bebês, os sujeitos resilientes eram temperamentalmente mais fáceis, de boa índole e afetuosos, com menos dificuldade para comer e dormir; na infância eram descritos como mais alertas, independentes, socialmente orientados e avançados na comunicação e na habilidade de auto-ajuda, tinham muitos interesses e se engajavam em atividades e passatempos que the proporcionavam alívio diante da adversidade e razão para se orgulhar. Hertzig constatou que as crianças resilientes estavam inseridas em famílias de quatro ou menos irmãos, com um intervalo de dois anos entre elas e seu irmão mais próximo, poucas tinham experimentado separações prolongadas dos primeiros cuidadores durante o primeiro ano de vida, e todas tinham estabelecido um vínculo afetivo forte durante a infância e anos pré-escolares.
O temperamento também pode ser considerado um fator de risco ao interagir com outros fatores (Strelau \& Eliasz, 1994). De acordo com Clarke e Clarke (1994) a irritabilidade temperamental, a falta de sociabilidade, a falta de segurança emocional e ligações afetivas fortes com alguma pessoa, a falta de apoio no ambiente escolar e uma situação social caracterizada pela pobreza e falta de esperança são os principais fatores de risco que predispõem o indivíduo à vulnerabilidade pessoal e à adversidade.

Considerado também como um fator de risco e incluído no modelo dos Cinco Grandes Fatores (CGF), o fator neuroticismo, conforme explica Nunes (2000), refere-se ao nível crônico de ajustamento emocional e instabilidade. Alto neuroticismo identifica indivíduos propensos a sofrimentos psicológicos e que podem apresentar altos níveis de ansiedade, depressão, hostilidade, vulnerabilidade, autocrítica e impulsividade.

O neuroticismo, acrescenta o autor, também inclui idéias não realísticas, baixa tolerância à frustração e respostas de coping não adaptativas, lembrando, também, que "baixos escores em neuroticismo não indicam necessariamente que o indivíduo tenha boa saúde mental, porém o que pode ser definido é que eles são simplesmente calmos, relaxados, estáveis, menos agitados" (Nunes, 2000, p.20).

Para o presente estudo, é importante salientar que o neuroticismo tem sido freqüentemente associado às dimensões de temperamento avaliadas pela escala PTS. De acordo com dados da literatura desse instrumento, considerando as definições teóricas envolvidas na definição e descrição das dimensões, e com base em estudos experimentais, são esperadas correlações negativas entre o neuroticismo e as dimensões força de excitação, força de inibição e mobilidade, presentes na escala PTS (Strelau et al., 1999).

Uma outra característica correlacionada negativamente ao neuroticismo, e levada em consideração no presente trabalho, é a auto-estima. A auto-estima está relacionada à saúde mental e ao bem-estar psicológico e a sua carência se relaciona com certos fenômenos mentais negativos como depressão e suicídio (Mruck, 1998).

Problemas sociais contemporâneos como abuso de drogas, gravidez precoce, fracasso escolar, delin- 
qüência, agressividade, distúrbios alimentares e dificuldades relacionais estão freqüentemente associados a esse constructo. Nesse sentido, a auto-estima é freqüentemente apontada na literatura como um aspecto relevante na prevenção de desajustamento psicossocial, em razão de seu efeito moderador sobre traços de personalidade, como neuroticismo, socialização, extroversão, e sobre questões desenvolvimentais, como rendimento escolar, identidade de gênero e resiliência. Entretanto poucos estudos empíricos são encontrados na literatura nacional sobre o tema, em parte, devido à indefinição conceitual e à complexidade da operacionalização desse constructo.

É importante salientar que o neuroticismo, por meio da dimensão denominada vulnerabilidade, está negativamente correlacionado à auto-estima, pois a escala de vulnerabilidade, conforme explica Hutz e Nunes (2001), avalia, entre outros aspectos, "quão intensamente as pessoas vivenciam sofrimentos em decorrência da aceitação dos outros para consigo"; nesse sentido "as pessoas que apresentam um escore muito alto nesse fator tendem a ter baixa auto-estima, relatam ter grande medo de que seus amigos os deixem em decorrência de seus erros (p.40)".

Considerando as relações que podem ser estabelecidas entre temperamento, neuroticismo e auto-estima e a importância dessas características para o ajustamento emocional e social do indivíduo, este estudo objetivou investigar padrões de correlação estabelecidos entre características individuais relacionadas ao temperamento, neuroticismo e auto-estima.

\section{Método}

\section{Participantes}

Participaram do presente estudo 42 universitários do curso de Psicologia de uma universidade do interior de São Paulo com idade entre 19 e 21 anos, sendo $36(85,7 \%)$ do sexo feminino e $6(14,3 \%)$ do sexo masculino.

\section{Instrumentos}

Foram utilizados os seguintes instrumentos:
1) Pavlovian Temperament Survey - versão adolescentes e adultos (Guzzo, Primi \& Ito, 2003; Guzzo, Riello \& Primi, 1996), constituída por 57 itens que avaliam três dimensões baseadas nas propriedades pavlovianas do sistema nervoso: força de excitação, que representa a capacidade do indivíduo manter-se sem inibição diante de uma situação de estimulação; força de inibição, que refere-se à capacidade do indivíduo interromper um determinado comportamento quando necessário ou efetuar uma mudança de reação e mobilidade, que está relacionada à habilidade do indivíduo de responder adequadamente a contínuas mudanças no ambiente.

2) Escala Fatorial de Ajustamento Emocional/ Neuroticismo (EFN) (Hutz e Nunes, 2001), composta por 82 itens que avaliam quatro dimensões: vulnerabilidade, constituída por itens que descrevem medo de críticas, insegurança, baixa auto-estima, dificuldade de tomar decisões, medo de abandono das pessoas mais próximas; desajustamento psicossocial, com itens que descrevem comportamentos sexuais de risco ou atípicos, adição ou consumo exagerado de álcool, hostilidade com pessoas ou animais, necessidade recorrente em chamar a atenção; ansiedade, que descreve sintomas somáticos relacionados com ansiedade, irritabilidade, transtornos do sono, impulsividade, sintomas de pânico, mudanças de humor; e depressão, que apresenta itens relacionados com escalas de depressão, suicídio e desesperança.

3) Escala de Auto-Estima de Rosenberg: trata-se de uma escala composta por 11 itens, destinada à avaliação da auto-estima por meio de uma única dimensão, tendo sido a versão brasileira do instrumento adaptada e validada por Hutz (s/d).

\section{Procedimentos}

Os dados foram coletados em aplicações coletivas em sala de aula, obedecendo à seguinte ordem de aplicação: 1) Escala Fatorial de Ajustamento Emocional/Neuroticismo, 2) Pavlovian Temperament Survey e 3) Escala de Auto-Estima de Rosenberg.

\section{Resultados}

Inicialmente apresentar-se-á os dados referentes às características de temperamento, ajustamento 
emocional/neuroticismo e auto-estima dos participantes e, em seguida, serão apresentados os coeficientes de correlação calculados por meio da correlação de Pearson, obtidos entre as dimensões avaliadas pelos instrumentos utilizados na pesquisa - Pavlovian Temperament Survey, Escala Fatorial de Ajustamento Emocional/Neuroticismo e a Escala de Auto-Estima de Rosenberg. Vale ressaltar que serão consideradas as correlações obtidas intra e inter instrumentos.

No que diz respeito às características de temperamento, avaliadas por meio da escala PTS, essa amostra apresentou um perfil de temperamento caracterizado por pontuações médias mais elevadas em mobilidade $(51,78)$ e força de inibição $(51,39)$ e menores em força de excitação $(41,93)$ (Tabela 1).

Resultados obtidos na EFN indicaram que os participantes obtiveram pontuações médias muito próximas nas quatro dimensões avaliadas: vulnerabilidade (VUL) (24,53), ansiedade (ANS) $(24,37)$, desajustamento psicossocial (DES PSIC) $(24,63)$ e depressão (DEP) (23,03). Já na Escala de Auto-Estima
(AE), a pontuação média obtida foi de 31,74 pontos (Tabela 1).

Apresentadas as características de temperamento, ajustamento emocional, neuroticismo e auto-estima da amostra, serão descritos a seguir os dados referentes às correlações obtidas entre as dimensões avaliadas pela Pavlovian Temperament Survey, Escala Fatorial de Ajustamento Emocional/Neuroticismo e a Escala de Auto-Estima de Rosenberg (Tabela 2).

Resultados obtidos indicaram correlações moderadas e significativas entre as várias dimensões avaliadas pelos três instrumentos. Considerando a escala PTS, foi possível constatar que a dimensão força de excitação, que se refere à capacidade do indivíduo manter-se sem inibição diante de uma situação de estimulação intensa e prolongada, apresentou correlação negativa significativa com as dimensões vulnerabilidade $(-0,403$ $p<0,05)$ e ansiedade $(-0,360 p<0,05)$, avaliadas pela EFN. A dimensão força de inibição, que se refere à capacidade do indivíduo interromper um determinado comportamento quando necessário ou efetuar uma mudança de

Tabela 1. Média e desvio-padrão obtidos nas escalas PTS, EFN e AE.

\begin{tabular}{|c|c|c|c|c|c|c|c|c|}
\hline & \multicolumn{5}{|c|}{ PTS } & \multicolumn{2}{|c|}{ EFN } & \multirow{2}{*}{$\begin{array}{l}A E \\
A E\end{array}$} \\
\hline & $\mathrm{FE}$ & $\mathrm{FI}$ & $\mathrm{MO}$ & VUL & ANS & DES PSIC & DEP & \\
\hline$M$ & 41,93 & 51,39 & 51,78 & 24,53 & 24,37 & 24,63 & 23,03 & 31,74 \\
\hline DP & 4,82 & 6,59 & 7,15 & 4,20 & 4,82 & 3,74 & 3,86 & 3,51 \\
\hline
\end{tabular}

M: Média; DP: Desvio-padrão; PTS: Pavlovian Temperament Survey; EFN: Escalas Fatorial de Ajustamento Emocional/Neuroticismo; AE: Auto-estima; FE: Força de excitação; FI: Força de inibição; MO: Mobilidade; VUL: Vulnerabilidade; ANS: Ansiedade; DES PSIC: Desapontamento psicossocial; DEP: Depressão.

Tabela 2. Indices de correlações entre a Pavlovian Temperament Survey (PTS), Escalas Fatorial de Ajustamento Emocional/Neuroticismo (EFN) e a Escala de Auto-estima de Rosenberg.

\begin{tabular}{|c|c|c|c|c|c|c|c|c|c|}
\hline & & \multicolumn{5}{|c|}{ PTS } & \multicolumn{2}{|c|}{ EFN } & \multirow{2}{*}{$\begin{array}{l}A E \\
A E\end{array}$} \\
\hline & & $\mathrm{FE}$ & $\mathrm{FI}$ & $\mathrm{MO}$ & VUL & ANS & DES PSIC & DEP & \\
\hline \multirow{5}{*}{ PTS } & $\mathrm{FE}$ & 1,000 & & & & & & & \\
\hline & $\mathrm{FI}$ & 0,202 & 1,000 & & & & & & \\
\hline & $\mathrm{MO}$ & 0,156 & $-0,196$ & 1,000 & & & & & \\
\hline & VUL & $-0,403^{*}$ & $-0,037$ & $-0,308$ & 1,000 & & & & \\
\hline & ANS & $-0,360^{*}$ & $-0,411^{*}$ & 0,292 & 0,360 & 1,000 & & & \\
\hline \multirow{2}{*}{ EFN } & DES PSIC & 0,055 & $-0,226$ & $0,383^{*}$ & 0,062 & $0,447^{* *}$ & 1,000 & & \\
\hline & DEP & $-0,137$ & $-0,050$ & $-0,041$ & $0,364^{*}$ & 0,310 & 0,043 & 1,000 & \\
\hline$A E$ & $\mathrm{AE}$ & 0,159 & 0,050 & 0,264 & $0,672^{* *}$ & $-0,405$ & $-0,283$ & $-0,378$ & 1,000 \\
\hline
\end{tabular}

${ }^{*} p<0,05 ;{ }^{* *} p<0,01$; AE: Auto-estima; FE: Força de excitação; FI: Força de inibição; MO: Mobilidade; VUL: Vulnerabilidade; ANS: Ansiedade; DES PSIC: Desapontamento psicossocial; DEP: Depressão. 
reação, apresentou correlação negativa significativa com a dimensão ansiedade $(-0,411 p<0,05)$. Já a dimensão mobilidade, que avalia a habilidade do indivíduo de responder adequadamente a contínuas mudanças no ambiente, se correlacionou positiva e significativamente com a dimensão desajustamento psicossocial (0,383 $p<0,05)$, e apresentou correlação negativa moderada com a dimensão vulnerabilidade (-0,308). Não foi observada nenhuma correlação moderada ou significativa entre as próprias dimensões avaliadas pela PTS e quando consideradas as dimensões avaliadas pela PTS e a Escala de Auto-Estima.

No que diz respeito às correlações intradimensões da EFN, foi possível constatar que: vulnerabilidade apresentou correlações positivas significativas com ansiedade $(0,360 p<0,05)$ e depressão $(0,364 p<0,05)$; desajustamento psicossocial se correlacionou positiva e significativamente com ansiedade $(0,447 p<0,01)$, já depressão e ansiedade são dimensões correlacionadas positiva e moderadamente $(0,310)$.

Quando consideradas as correlações obtidas entre EFN e a Escala de Auto-Estima, contatou-se que a auto-estima está correlacionada negativa e significativamente com vulnerabilidade $(-0,672 p<0,05)$, e negativa e moderadamente com ansiedade $(-0,405)$ e depressão $(-0,378)$

\section{Discussão}

Resultados obtidos na caracterização do temperamento indicaram que essa amostra apresentou como características predominantes de temperamento, respectivamente, a mobilidade e a força de inibição; a força de excitação apareceu como uma característica menos presente. Comparando esse perfil de temperamento aos dados obtidos por Riello (1999) em sua pesquisa envolvendo 135 participantes de 12 a 21 anos, divididos em três grupos com diferentes graus de envolvimento com a prática da natação, constatou-se um perfil semelhante, predomínio da dimensão de mobilidade, seguida por força de inibição e por último força de excitação. Resultados similares são obtidos por Guzzo et al. (2003) no estudo de normatização da escala para a realidade brasileira, que envolveu a participação de 952 adolescentes der 14 a 18 anos e por Strelau et al. (1999) nas pesquisas transculturais desenvolvidas com a PTS envolvendo resultados coletados em 16 países. Um perfil de temperamento um pouco diferenciado foi obtido por Ito, Gobitta e Guzzo (2003) em uma pesquisa envolvendo 161 participantes de 19 a 53 anos, os quais apresentaram um perfil no qual predominava a força de inibição seguida pela mobilidade e, por último, como nos estudos anteriores, a força de excitação.

Essa diferenciação no perfil de temperamento envolvendo as dimensões $\mathrm{MO}$ e Fl encontram respaldo na literatura (Strelau et al., 1999), que afirma que são esperadas variações nas pontuações das dimensões aproximadamente aos 30 anos, existindo uma tendência de as pontuações de força de excitação e mobilidade diminuírem com o passar dos anos e de as pontuações de força de inibição aumentarem. Tal tendência é justificada pelos autores devido ao ambiente e à experiência influenciarem a expressão das características temperamentais.

Como foi possível constatar acima, os participantes do estudo de Ito, Gobitta e Guzzo (2003) encontram-se numa faixa etária superior a dos participantes envolvidos nesta pesquisa, e a dos envolvidos nos estudos de Riello (1999) e Guzzo et al. (2003).

No que diz respeito às médias obtidas nos fatores avaliados pela PTS, constatou-se que os valores não apresentaram grandes variações. Guzzo et al. (2003) obtiveram pontuações médias de $\mathrm{MO}=50,63, \mathrm{Fl}=50,13$ e $F E=41,46$; nos resultados obtidos por Riello (1999), as médias obtidas pelos três grupos estudados variaram em MO de 50,07 a 51,82, em FI de 48,98 a 49,44 e em FE de 41,16 a 41,72. Considerando as idades similares dos participantes envolvidos nesses estudos, tal proximidade nas médias obtidas nas dimensões entre os diferentes estudos também pode ser justificada pelas alterações na expressão do temperamento com o passar dos anos, fruto da influência da experiência e do contexto.

Considerando as normas estabelecidas no estudo da PTS por Guzzo et al. (2003), as pontuações médias obtidas pelos participantes deste estudo situam-nos dentro da média quando comparados a uma amostra brasileira mais ampla, o percentil obtido para mobilidade foi de 51\%, em força de inibição 50\% e em força de ecxitação 49\% .

De modo geral, as pontuações médias obtidas nas dimensões avaliadas pela PTS sugerem que os participantes estudados apresentariam uma tendência 
em: apresentar reações adequadas perante mudanças inesperadas, adaptar-se facilmente a pessoas, situações e contextos, controlar a expressão de reações emocionais e comportamentos, evitar situações de alto valor estimulativo.

No que diz respeito às características de ajustamento emocional/neuroticismo, pontuações médias obtidas pelos participantes foram bastante próximas nas quatro dimensões avaliadas. Comparando os resultados obtidos às normas estabelecidas para o instrumento, em estudo que envolveu a participação de 1.176 pessoas, de ambos os sexos, com idade entre 16 e 28 anos ou mais (Hutz \& Nunes, 2001), constatou-se que na dimensão vulnerabilidade a média obtida situa os participantes entre o percentil 45 e 50. Da mesma forma, em desajustamento psicossocial o percentil constatado situa os participantes entre o percentil 55 e 60 e nas dimensões ansiedade e depressão o percentil foi 50 e 40, respectivamente.

Esses dados permitem verificar que essa amostra, quando comparada às normas, apresentou um índice de desajustamento social um pouco maior e um índice de depressão um pouco menor. Ansiedade e vulnerabilidade encontram-se na média.

A auto-estima dos participantes, avaliada pela Escala de Auto-Estima de Rosenberg, apresentou pontuação média elevada quando comparada à média obtida por Hutz (s/d) junto a uma amostra constituída por 460 participantes, a qual apresentou uma média de 18,66. A partir desse dado constatou-se que os participantes deste estudo tendem a apresentar uma auto-estima favorável.

Resumidamente, considerando as normas e médias brasileiras estabelecidas para a PTS, EFN e Escala de Auto-Estima, constatou-se que o grupo de participantes deste estudo apresenta pontuações consideradas na média nas dimensões de temperamento e ajustamento emocional/neuroticismo e mais favoráveis em auto-estima.

Os resultados referentes às correlações encontradas entre as dimensões que compõem os três instrumentos bem como entre as próprias dimensões da PTS e da EFN serão analisados a seguir.

As três dimensões avaliadas pela PTS apresentaram correlações significativas com três dimensões da EFN, força de excitação e força de inibição), se correlacionaram negativamente com ansiedade, e ainda, força de excitação também apresentou correlação negativa com vulnerabilidade. Mobilidade apresentou correlação positiva com a dimensão desajustamento psicossocial da EFN.

De acordo com dados da literatura (Strelau et al., 1999), são esperadas correlações negativas entre os três fatores avaliados pela PTS e o neuroticismo, indivíduos com altas pontuações em força de excitação, força de inibição e Mobilidade tenderiam a apresentar baixas pontuações em neuroticismo e indivíduos com baixas pontuações em força de excitação, força de inibição e Mobilidade apresentariam pontuações elevadas em neuroticismo.

O neuroticismo, de acordo com Costa e McCrae, é uma dimensão da personalidade caracterizada por uma tendência do indivíduo a experienciar emoções negativas as quais inclui as facetas de ansiedade, hostilidade, depressão, baixa auto-estima, impulsividade e manifesta-se numa tendência geral para experienciar afetos negativos semelhantes a medo, tristeza, embaraço, raiva, culpa, repugnância; seus rompantes emocionais interferem na adaptação; altas pontuações em neuroticismo predispõem o indivíduo a idéias irracionais e a menor controle dos impulsos e apresentação de respostas de coping menos adaptativas (Angleitner, Kohnstamm, Slotboom \& Besevegis, 1998).

Como apresentado na descrição da Pavlovian Temperament Survey, a força de excitação está relacionada à habilidade do indivíduo de resistir a uma estimulação intensa e prolongada sem apresentar inibição, e em uma de suas facetas se manifesta como ausência de alterações ou distúrbios emocionais no desempenho de atividade sob pressão física e/ ou social, comparada à descrição de neuroticismo.

A mobilidade é definida em linhas gerais como a habilidade de responder adequadamente às mudanças do ambiente e em vários estudos aparece correlacionada à força de excitação; e a força de inibição, como a capacidade do indivíduo de interromper um determinado comportamento quando necessário, ou efetuar uma mudança de reação (Strelau et al., 1999).

Considerando as definições apresentadas anteriormente e o fato de os indivíduos com altas pontuações em FE, FI e MO apresentarem características 
opostas às apresentadas por indivíduos com altas pontuações em neuroticismo, são esperadas correlações negativas entre a PTS e a EFN.

De fato, dados empíricos coletados em 14 estudos envolvendo amostras americanas, alemãs e polonesas apóiam as hipóteses teóricas anteriormente levantadas, ou seja, as três escalas da PTS apresentam correlações negativas com o fator neuroticismo avaliado pelo EPQ-R de Eysenck, Eysenck e Barrett e pelos três inventários NEO de Costa e McCrae. A correlação média constatada nesses estudos foi: FE $(-0,455)$, Fl $(-0,32)$ e MO $(-0,39)$ (Strelau et al., 1999).

No Brasil, estudos sobre (Rodrigues, 2001, Geller, 2000, Silva, 1999) a relação entre força de excitação e ansiedade traço (La Rosa, 1993, Strelau et al., 1999) constataram que indivíduos com pontuações elevadas em força de excitação do sistema nervoso apresentam baixos níveis de ansiedade traço, sendo portanto estabelecidas correlações negativas entre esses constructos.

Comparando os dados da literatura apresentados e as correlações obtidas entre a PTS e o EFN, foi possível constatar que essas estão de acordo com o esperado no que diz respeito a FE e Fl; em MO, no entanto, o mesmo não ocorreu, pois foi constatada uma correlação positiva entre essa dimensão e o desajustamento psicossocial. Uma hipótese que pode ser levantada com relação a essa correlação não esperada está relacionada à desejabilidade social; os participantes do estudo podem ter respondido aos itens dessas duas dimensões de acordo com o que acreditariam ser esperado socialmente, ambas as dimensões descrevem como o indivíduo está adaptado ao ambiente.

Neste estudo não foram constatadas correlações significativas entre as dimensões força de excitação, força de inibição e mobilidade avaliadas pela PTS. Strelau et al. (1999) afirmam que as dimensões avaliadas não são ortogonais, no entanto são esperadas correlações positivas elevadas entre força de excitação e mobilidade, fato não constatado, o que pode ser mais um indício de que os indivíduos responderam aos itens de mobilidade de acordo com o que seria socialmente esperado.

No que diz respeito às correlações obtidas entre as próprias dimensões avaliadas pela EFN, resultados obtidos indicaram correlações positivas entre ansiedade e as dimensões vulnerabilidade, desajustamento psicossocial e depressão e entre depressão e vulnerabilidade. De acordo com Nunes (2000) são esperadas correlações positivas entre as quatro dimensões avaliadas pela EFN, fato comprovado em sua pesquisa numa amostra brasileira envolvendo 792 participantes, a qual obteve os seguintes coeficientes de correlação significativos ( $p<0,01)$ : vulnerabilidade e desajustamento psicossocial $=0,25$; vulnerabilidade e ansiedade $=0,56$; vulnerabilidade e depressão=0,60; desajustamento psicossocial e ansiedade $=0,36$; desajustamento psicossocial e depressão=0,28; ansiedade e depressão =0,48. Como afirma esse autor,"o padrão de correlações obtidas tem um sentido teórico, uma vez que confirma adequadamente a relação entre traços de personalidade que descrevem diferentes níveis de depressão, ansiedade, vulnerabilidade e padrões de desadaptação" (Nunes, 2000, p.32).

A correlação entre a Escala de Auto-Estima de Rosenberg e a EFN apresentou correlações negativas entre auto-estima e vulnerabilidade, ansiedade e depressão. A esse respeito, Nunes (2000) relata que elevadas correlações negativas entre a Escala de Auto-Estima de Rosenberg e o neuroticismo são esperadas, pois pessoas altas em neuroticismo tendem a ter uma avaliação depreciativa de si mesmo, pois tendem a ter altos níveis de depressão, de vulnerabilidade e ansiedade. Em sua pesquisa, o autor citado obteve correlações negativas significativas $(p<0,01)$ entre auto-estima e vulnerabilidade $(-0,62)$, auto-estima e ansiedade $(-0,41)$ e auto-estima e depressão $(-0,66)$.

Comparando os dados deste estudo com os de Nunes (2000), foi possível observar que são similares, tendo sido constatadas correlações entre as mesmas dimensões. Tais dados apresentam-se favoráveis e confirmam a validade de constructo da EFN, investigada anteriormente no estudo de Nunes (2000).

No que diz respeito às correlações obtidas entre a Escala de Auto-Estima de Rosenberg e a PTS, foram obtidas correlações baixas e positivas. Não foram encontrados na literatura estudos envolvendo correlações entre as dimensões avaliadas pela PTS e a auto-estima. No entanto, considerando as definições e as descrições dos constructos, bem como o padrão de correlação esperado e obtido entre PTS e EFN e EFN e auto-estima, seriam esperadas correlações positivas 
entre força de excitação, força de inibição e mobilidade e auto-estima. São necessários novos estudos para investigar melhor as correlações entre este temperamento e auto-estima.

De acordo com os dados da literatura e os padrões de correlação encontrados, indivíduos com pontuações elevadas em força de excitação, força de inibição e mobilidade tenderiam a apresentar elevada auto-estima e baixos índices de vulnerabilidade, desajustamento psicossocial, ansiedade e depressão. Da mesma forma, indivíduos com pontuações baixas em força de excitação, força de inibição e mobilidade tenderiam a apresentar baixa auto-estima e elevados índices de vulnerabilidade, desajustamento psicossocial, ansiedade e depressão.

Os dados apresentados neste estudo permitem verificar, de forma preliminar, como diferentes características de personalidade se relacionam, que perfil individual predispõem e qual o tipo de interação que favorecem com o ambiente. Informações sobre as diferenças individuais, perfis de personalidade e a influência exercida por essas na interação do indivíduo com o meio, bem como a influência que o meio exerce no indivíduo, são dados importantes na elaboração e implementação de programas de prevenção e intervenção.

É importante lembrar que estudos correlacionais também são utilizados na investigação da validade de constructo convergente e discriminante dos testes psicológicos, os quais estão embasados na premissa de que o constructo apresenta correlações positivas com variáveis com as quais ele deve teoricamente correlacionar-se (validade convergente) e não apresente correlações positivas significativas com variáveis das quais ele deve diferir (validade discriminante) (Anastasi \& Urbina, 2000).

Vale ressaltar que é preciso certa cautela na generalização desses resultados, sendo necessária a realização de novos estudos que envolvam maior número de participantes, de diferentes idades e contextos, para confirmar os dados obtidos.

\section{Referências}

Anastasi, A., \& Urbina, S. (2000). Testagem psicológica. Porto Alegre: ArtMed.
Angleitner, A., Kohnstamm, G. A., Slotboom, A. M., \& Besevegis, E. (1998). Emotional stability: developmental perspectives from childhood to adulthood. In G. A. Kohnstamm, C. F. Halverson Jr., I. Mervielde \& V. L. Havill (Orgs.), Parental descriptions of child personality (pp.85-103). New Jersey: Lawrence Erlbaum Associates.

Brito, R. C. E., \& Koller, S. H. (1999). Desenvolvimento humano e redes de apoio social e afetivo. In A. M. Carvalho (Org.), O mundo social da criança: natureza e cultura em ação (pp.115-126). São Paulo: Casa do Psicólogo.

Bronfenbrenner, U. (1995). Developmental ecology through space and time: a future perspective. In P. Moen, G. H. Elder Jr. \& K. Lüscher (Orgs.), Examining lives in context (pp.619-647). Washington, DC: American Psychological Association.

Buss, A. H. (1995). Personality: temperament, social behavior, and the self. Massachusetts: Allyn and Bacon.

Clark, L. A., \& Watson, D. (1999). Temperament: a new paradigm for trait psychology. In L. A. Pervin \& O. P. John (Orgs.), Handbook of personality: theory and research (pp.399-423). New York: Guilford.

Clarke, A. M., \& Clarke, A. D. B. (1994). Variations, deviations, risks, and uncertainties in human development. In W. B. Carey \& S. C. McDevitt (Orgs.), Prevention and early intervention: individual differences as risk factors for the mental health of children (pp.83-91). New York: Brunner/ Mazel.

Chess, S., \& Thomas, A. (Eds.) (1987). Annual progess in child psychiatry and child development. New York: Brunner/ Mazel.

Digman, J. M. (1990). Personality structure: emergence of the five-factor model. Annual Review of Psychology, 41, 417-440

Eysenck, H. J. (1974). A desigualdade do homem. Rio de Janeiro:Zahar.

Geller, C. A. (2000). A relação das peculiaridades tipológicas do sistema nervoso com o nível de ansiedade: traço em estudantes de segundo grau. Dissertação de mestrado não-publicada, Universidade Federal de Santa Maria.

Goldsmith, H. H., Buss, A. H., Plomin, R., Rothbart, M. K., Chess, S. Thomas, A. , Hinde, R. A., \& McCall, R. B. (1987). Rountdtable: what is temperament? Four approaches. Child Development, 58, 505-529.

Graziano, W. G., Jensen-Campbell, L. A., \& Sullivan-Logan, G. M. (1998). Temperament, activity, and expectations for later personality development. Journal of Personality and Social Psychology, 74 (5), 1266-1277.

Guzzo, R. S. L., Primi, R., \& Ito, P. C. P. (2003). PTS - Pavlovian Temperament Survey, versão adolescente adulto: consistência interna e normatização para a realidade brasileira. Estudos de Psicologia (UFRN), 8 (3), 525-533.

Guzzo, R. S. L., Riello, I. C., \& Primi, R. (1996). Pavlovian Temperament Survey - PTS: análise de itens e teste de realidade. Psicologia Escolar e Educacional, 1 (1), 53-59.

Hertzig, M. E. (1994). Conditions of risk for maldevelopment: prematuriry. In W. B. Carey \& S. C. McDevitt (Orgs.), Prevention and Early intervention: individual differences as 
risk factors for the mental health of children (pp.92-102). New York: Brunner/Mazel.

Hutz, C. S. (s/d). Escala de Auto-Estima de Rosemberg. Acessado em junho, 2002, disponível em: http://www.psicologia. ufrgs.br/laboratorio/rosenb.htm

Hutz, C. S., \& Nunes, C. H. S. S. (2001). Escala Fatorial de Ajustamento Emocional/Neuroticismo (EFN). São Paulo: Casa do Psicólogo.

Hutz, C. S., Nunes, C. H., Silveira, A. B., Serra, J., Anton, M., \& Wieczorek, L. S. (1998). O desenvolvimento de marcadores para avaliação da personalidade no modelo dos cinco grandes fatores. Psicologia: Reflexão e Crítica, 11 (2), 395-409.

Ito, P. C. P., Gobitta, M., \& Guzzo, R. S. L. (2003). Características de temperamento em diferentes áreas de atuação: estudo preliminar. Manuscrito não-publicado.

La Rosa, J. (1993). Inventário de ansiedade traço - estado: características psicométricas. Estudos de Psicologia (Campinas), 10 (2), 81-92.

Losel, F., \& Bliesener, T. (1994). Some high-risk adolescents do not develop conduct problems: a study of protective factors. International Journal of Behavioral Development, $17(4), 753-777$.

Mruck, C. (1998) .Auto-Estima:Investigación, teoríay práctica. Bilbao: Desclée de Brouwer.

Nunes, C. H. S. (2000). A construção de um instrumento de medida para o fator neuroticismo/ estabilidade emocional dentro do modelo de personalidade dos cinco grandes fatores. Dissertação de mestrado não-publicada, Universidade Federal do Rio Grande do Sul, Porto Alegre.

Riello, I. C. (1999). Temperamento: perfil de adolescentes com diferentes competências em natação. Tese de doutorado não-publicada, Pontifícia Universidade Católica de Campinas.

Rodrigues, A. A. (2001). Voleibol feminino: uma análise da ansiedade traço influenciada pelas forças do processo de excitação do sistema nervoso. Dissertação de mestrado não-publicada, Universidade de São Paulo.
Rothbart, M. K. (1986). A psychobiological approach to the study of temperament. In G. A. Kohnstamm (Org.), Temperament discussed: temperament and development in infancy and childhood (pp.63-72). Lisse: Swets \& Zeitlinger.

Rothbart, M. K., \& Ahadi, S. A. (1994). Temperament and the development of personality. Journal of Abnormal Psychology, 103 (1), 55-66.

Silva, M. M. (1999). Um estudo da diferença entre níveis de ansiedade de traço de indivíduos de ambos os sexos, diferenciados pelas peculiaridades tipológicas dos sistema nervoso. Dissertação de mestrado não-publicada, Universidade Federal de Santa Maria.

Strelau, J. (1998). Temperament: a psychological perspective. New York: Plenum Press.

Strelau, J., Angleitner, A., \& Newberry, B. H. (1999). Pavlovian Temperament Survey (PTS) An International Handbook. Seattle: Hogrefe \& Huber Publishers.

Strelau, J., \& Eliasz, A. (1994). Temperament riskfactors for type A behavior pattern in adolescence. In W. B. Carey \& S. C. McDevitt (Orgs.), Prevention and Early intervention: individual differences as risk factors for the mental health of children (pp.42-49). New York: Brunner/Mazel.

Szymanski, H., \& Yunes, M. A. M. (2001). Resiliência: noção, conceitos afins e considerações críticas. In J. Tavares, M. A. M. Yunes, H. Szymanski, A. M. S. Pereira, H. Ralha-Simões \& M. A. C. D. Castro (Orgs.), Resiliência e educação (pp.13-42). São Paulo: Cortez Editora.

Teglasi, H. (1995). Assessment of temperament. Eric Digest. Available from: http://www.ed.gov/databases/ericdigest/ed389963.html

Teglasi, H. (1998). Introduction to the mini-series: implications of temperament for the practice of school Psychology. School Psychology Review, 24 (4), 475-478.

Recebido em: 6/9/2006

Aprovado em: 24/10/2006 
